International Journal of Modern Physics A

(C) World Scientific Publishing Company

\title{
R-Parity Violation and Family Symmetry *
}

\author{
YUJI KAJIYAMA \\ National Institute of Chemical Physics and Biophysics, \\ Ravala 10, Tallinn 10143, Estonia \\ yuji.kajiyama@kbfi.ee
}

Received 15 June 2007

\begin{abstract}
In this talk, we investigate the implications of R-parity violating (RPV) operators in a model with family symmetry 1 . Family symmetry can determine the form of RPV operators as well as the Yukawa matrices. We consider a concrete model with non-abelian discrete symmetry $Q_{6}$, which has only three RPV trilinear operators with no baryon number violating terms. We find that ratios of decay rates of the lepton flavor violating processes are fixed thanks to the family symmetry, predicting $B R(\tau \rightarrow 3 e) / B R(\tau \rightarrow$ $3 \mu) \sim 4 m_{\mu}^{2} / m_{\tau}^{2}$.

Keywords: Supersymmetric models; Flavor symmetries; Masses and mixing.

PACS numbers: 12.60.Jv, 11.30.Hv, 12.15.Ff, 14.60.Pq
\end{abstract}

\section{Introduction}

Despite the remarkable success of the gauge sector of the Standard Model (SM), there still exist some problems in the Higgs and Yukawa sectors. The Yukawa matrices are responsible for the masses and mixings of matter fermions: quarks and leptons. The Yukawa sector in the SM can give experimentally consistent masses and mixings, because it contains more free parameters than the number of observables in general. There is no predictivity in the Yukawa sector because of this redundancy of the parameters. One of the ideas to overcome this issue is to introduce a family symmetry (flavor symmetry), which is the symmetry between generations. In this paper we consider a concrete model which is symmetric under the binary dihedral group $Q_{6}[23$.

On the other hand, in the Higgs sector, the most important problem is that the Higgs boson has not been experimentally discovered yet. Discovery of the Higgs boson is expected at the Large Hadron Collider (LHC). In the SM, the Higgs mass is quadratic divergent. This problem is solved by introducing Supersymmetry (SUSY) at $O(1) \mathrm{TeV}$. The Minimal Supersymmetric Standard Model (MSSM) has low en-

* To appear in the proceedings of CTP symposium on Supersymmetry at LHC: Theoretical and Experimental Perspectives, The British University in Egypt, Cairo, Egypt, 11-14 March 2007. 
ergy SUSY. In general it contains gauge symmetric, lepton and baryon number violating operators

$$
W_{\mathbb{R}}=\frac{1}{2} \lambda_{i j k} L_{i} L_{j} E_{k}^{c}+\lambda_{i j k}^{\prime} L_{i} Q_{j} D_{k}^{c}+\frac{1}{2} \lambda_{i j k}^{\prime \prime} U_{i}^{c} D_{j}^{c} D_{k}^{c}+\mu_{i} H_{u} L_{i}
$$

in addition to the usual Yukawa couplings and $\mu$-term. The asymmetric properties $\lambda_{i j k}=-\lambda_{j i k}$ and $\lambda_{i j k}^{\prime \prime}=-\lambda_{i k j}^{\prime \prime}$ mean that $9+27+9+3=48$ (complex) parameters are included in this interactions. These couplings generate unacceptable processes such as Lepton Flavor Violating (LFV) processes and proton decay. The conservation of R-parity 89

$$
R=(-1)^{3 B+L+2 s},
$$

where $B, L$ and $s$ denote baryon, lepton number and spin of the particles, respectively, is one possibility to forbid the couplings Eq.(1). From the definition, R-parity is +1 for all SM particles and -1 for all their superpartners. However, R-parity is not the only possible choice to forbid the interactions. Matter- or lepton- and baryonparity 10 can be also a possibility. On the other hand, without R-parity, these coupling constants have to be strictly constrained not to conflict with experimental data. Constraints on the R-parity violating couplings have been obtained by many authors from LFV processes 11|12|13|14|15|16, neutrino mass 11|17|18|19|20, neutral meson system $21|22| 23|24| 25|26| 27|28| 29$, proton decay $13|30| 31$, and so on $9|32| 35$.

Family symmetries also constrain the form of R-parity interactions $19|30| 33 \mid 34$ as well as the Yukawa matrices. In the model that we consider $2\left[3\right.$, the $Q_{6}$ family symmetry reduces the 45 trilinear couplings to three: $\lambda, \lambda_{1}^{\prime}$ and $\lambda_{2}^{\prime}$. The baryon number violating couplings $\lambda_{i j k}^{\prime \prime} U_{i}^{c} D_{j}^{c} D_{k}^{c}$ are forbidden by the symmetry in our model, so it is guaranteed by the symmetry that the R-parity violating operators do not induce proton decay.

In this paper, we study the phenomenology of the three R-parity violating interactions in the model with $Q_{6}$ family symmetry 23 . First, we obtain upper bounds on three coupling constants $\lambda$ and $\lambda_{1,2}^{\prime}$ from the experimental constraints. Next we focus on LFV processes induced by $\lambda$. The $\lambda L L E^{c}$ coupling generates the LFV decays $\ell_{m}^{-} \rightarrow \ell_{i}^{-} \ell_{j}^{-} \ell_{k}^{+}(m, i, j, k$ denote the flavor of the charged lepton) at tree level, and the Branching Ratios $(B R)$ of the decay processes are proportional to $\lambda^{4}$. Therefore, the ratios of these processes are independent of $\lambda$ and can be predicted unambiguously to be $B R(\tau \rightarrow$ eee $) / B R(\tau \rightarrow \mu \mu \mu) \sim 4 m_{\mu}^{2} / m_{\tau}^{2}$. It reflects the properties of the family symmetry. We introduce the $Q_{6}$ symmetric model in the next section, and derive the predictions in the Sec. 3. Sec. 4 is conclusions.

This talk is based on ref. 1 . 


\section{The Model}

\subsection{Group Theory of $Q_{6}$ and Assignment}

The binary dihedral group $Q_{N}(N=2,4,6, \ldots)$ is a finite subgroup of $S U(2)$ and defined by the following set of $2 N$ elements

$$
Q_{N}=\left\{1, A, A^{2}, \ldots, A^{N-1}, B, A B, \ldots, A^{N-1} B\right\},
$$

where two dimensional representation of matrix $A$ and $B$ is given by

$$
A=\left(\begin{array}{cc}
\cos \phi_{N} & \sin \phi_{N} \\
-\sin \phi_{N} & \cos \phi_{N}
\end{array}\right), \phi_{N}=\frac{2 \pi}{N}, \quad B=\left(\begin{array}{cc}
i & \\
-i
\end{array}\right) .
$$

In this note, we consider the case of $N=6$ a.

$Q_{6}$ group contains 2 two-dimensional irreducible representations (irreps), $\mathbf{2}_{1}, \mathbf{2}_{2}$ and 4 one-dimensional ones $\mathbf{1}_{+, 0}, \mathbf{1}_{+, 2}, \mathbf{1}_{-, 1}, \mathbf{1}_{-, 3}$, where $\mathbf{2}_{1}$ is pseudo real and $\mathbf{2}_{2}$ is real representation. In the notation of $\mathbf{1}_{ \pm, n}(n=0,1,2,3), \pm$ stands for the change of sign under the transformation by matrix $A$, and $n$ the factor $\exp (i n \pi / 2)$ by $B$. So $\mathbf{1}_{+, 0}$ and $\mathbf{1}_{+, 2}$ are real representations, while $\mathbf{1}_{-, 1}$ and $\mathbf{1}_{-, 3}$ are complex conjugate to each other.

We consider a extension of Supersymmetric Standard Model with $Q_{6}$ family symmetry, where three generations of matter and Higgs fields are assumed to be embedded into two- and one- dimensional irreps. of $Q_{6}$ group. Assignment of $Q_{6}$ group for the quark, lepton and Higgs chiral supermultiplets are shown below in an obvious notation:

$$
\begin{aligned}
\mathbf{2}_{1}: Q_{I}, \\
\mathbf{2}_{2}: U_{I}^{c}, D_{I}^{c}, \hat{L}_{I}, E_{I}^{c}, N_{I}^{c}, H_{I}^{u}, \hat{H}_{I}^{d}, \\
\mathbf{1}_{+, 0}: L_{3}, E_{3}^{c}, \\
\mathbf{1}_{+, 2}: Q_{3}, Y, \\
\mathbf{1}_{-, 1}: U_{3}^{c}, D_{3}^{c}, H_{3}^{u}, H_{3}^{d}, \\
\mathbf{1}_{-, 3}: N_{3}^{c} .
\end{aligned}
$$

The generation indices $I, J, \ldots=(1,2)$ are applied to the $Q_{6}$ doublet, and $i, j, \ldots=$ $(1,2,3)$ to three generations throughout the paper. $Y$ is gauge singlet Higgs supermultiplet to give neutrino mass by seesaw mechanism. In a model without R-parity conservation, there is no distinction between lepton doublet and down type Higgs doublet, because both have the same gauge quantum numbers. In our model, $L_{3}$ and $H_{3}^{d}$ are distinguishable because they have different $Q_{6}$ quantum number to each other, although $L_{I}$ and $H_{I}^{d}$ belong to the same irreps. Therefore, we have written these fields as $\hat{L}_{I}, \hat{H}_{I}^{d}$, and physical lepton doublet and down type Higgs doublet $\left(L_{I}, H_{I}^{d}\right)$ should be written as linear combination of these.

${ }^{\mathrm{a}}$ In $Q_{8}$ model of re $\frac{36}{}$, definition of $Q_{N}$ is different from ours. Our $Q_{N}$ is equivalent to their $Q_{2 N}$. 


\subsection{Fermion mass matrices and diagonalization}

We assume that physical Higgs fields acquire complex vacuum expectation values (VEVs) $\left\langle H_{I}^{u, d}\right\rangle=v_{D}^{u, d} e^{i \theta^{u, d}} / 2$ and $\left\langle H_{3}^{u, d}\right\rangle=v_{3}^{u, d} e^{i \theta_{3}^{u, d}} / \sqrt{2}$ in order to avoid SUSY CP problem in soft SUSY breaking sector.

The quark mass matrices are given by

$$
\mathbf{m}^{u}=m_{t}\left(\begin{array}{ccc}
0 & q_{u} / y_{u} & 0 \\
-q_{u} / y_{u} & 0 & b_{u} \\
0 & b_{u}^{\prime} & y_{u}^{2}
\end{array}\right)
$$

and similarly for $\mathbf{m}^{d}$, where phases from VEVs have been absorbed into a part of the CKM matrix.

Many set of the parameters give observables consistent with experimental data, and one example is

$$
\begin{aligned}
& \theta_{q}=\theta_{3}^{d}-\theta^{d}-\theta_{3}^{u}+\theta^{u}=-1.25, q_{u}=0.0002150, b_{u}=0.04440, b_{u}^{\prime}=0.09300 \\
& y_{u}=0.99741, q_{d}=0.005040, b_{d}=0.02500, b_{d}^{\prime}=0.7781, y_{d}=0.7970 .
\end{aligned}
$$

One can easily obtain unitary matrices in explicit form which diagonalize mass matrices from these parameters. Moreover, since the CKM parameters and the quark masses are related to each other because of the family symmetry, we find that nine independent parameters (Eq.(77) ) of the model can well describe ten physical observables: there is one prediction. An example of the prediction is $\left|V_{t d} / V_{t s}\right|$, whose experimental value has been obtained from the measurement of the mass difference $\Delta m_{B_{s}}$ of the $B_{s}^{0}$ meson 6 .

$$
\begin{aligned}
& \text { Model : }\left|V_{t d} / V_{t s}\right|=0.21-0.23, \\
& \text { Exp. : }\left|V_{t d} / V_{t s}\right|=0.208_{-0.002}^{+0.001}{ }^{(\text {exp. })}{ }_{-0.006}^{+0.008} \text { (theo.). }
\end{aligned}
$$

The mass matrix in the charged lepton sector is:

$$
\mathbf{m}^{e}=\frac{1}{2}\left(\begin{array}{ccc}
-Y_{c}^{e} & Y_{c}^{e} & Y_{b}^{e} \\
Y_{c}^{e} & Y_{c}^{e} & Y_{b}^{e} \\
Y_{b^{\prime}}^{e} & Y_{b^{\prime}}^{e} & 0
\end{array}\right) v_{D}^{d} e^{-i \theta^{d}}
$$

One finds that $U_{e L}$ and $U_{e R}$ can be approximately written as

$$
U_{e L} \simeq\left(\begin{array}{ccc}
\epsilon_{e} & 1 / \sqrt{2} & 1 / \sqrt{2} \\
-\epsilon_{e} & -1 / \sqrt{2} & 1 / \sqrt{2} \\
1 & -\sqrt{2} \epsilon_{e} & 0
\end{array}\right), U_{e R} \simeq\left(\begin{array}{ccc}
0 & -1 & 0 \\
1 & 0 & \epsilon_{\mu} \\
-\epsilon_{\mu} & 0 & 1
\end{array}\right) e^{i \theta^{d}},
$$

where terms of $\mathcal{O}\left(\epsilon^{2}\right)$ are neglected, and small parameters $\epsilon_{e}, \epsilon_{\mu}$ are defined as

$$
\epsilon_{e}=\frac{m_{e}}{\sqrt{2} m_{\mu}}=3.42 \times 10^{-3}, \epsilon_{\mu}=\frac{m_{\mu}}{m_{\tau}}=5.94 \times 10^{-2} .
$$

The upper-right $2 \times 2$ block of $U_{e L}$ is the origin of maximal mixing of the atmospheric neutrino oscillation. 
As for the neutrino sector, we assume that a see-saw mechanism 7 takes place. However, we do not present the details of the neutrino sector here because there is no need to know it in the following analysis. We obtain some specific predictions of our model: (i) only an inverted mass hierarchy $m_{\nu_{3}}<m_{\nu_{1}}, m_{\nu_{2}}$ is consistent with the experimental constraint $\left|\Delta m_{21}^{2}\right|<\left|\Delta m_{23}^{2}\right|$, (ii) the $(e, 3)$ element of the MNS matrix is given by $\left|U_{e 3}\right| \simeq \epsilon_{e}$. See Refs. $\frac{3 \mid 4}{43}$ for details.

\section{R-Parity Violation}

Since $Q_{6}$ family symmetry controls the whole flavor structure of the model, the form of the R-parity violating couplings are also constrained by the family symmetry. We find that only possible trilinear couplings allowed by the symmetry can be written as

$$
W_{\text {R }}=\lambda L_{3} L_{I} E_{I}^{c}+\lambda_{1}^{\prime} L_{I}\left(i \sigma^{2}\right)_{I J} Q_{3} D_{J}^{c}+\lambda_{2}^{\prime} L_{I}\left(\sigma^{1}\right)_{I J} Q_{J} D_{3}^{c}
$$

in the physical lepton doublet $L_{I}$. Here the superpotential $W_{\not R}$ is written in the flavor eigenstates, so the unitary matrices $U_{u(L, R)}, U_{d(L, R)}$ and $U_{e(L, R)}$ should appear when we rotate the fermion components into their mass eigenstates. On the other hand, these matrices do not appear from sfermion components, because we approximate that sfermions are in the mass eigenstate basis. This approximation is valid in our model, because scalar masses in the soft SUSY breaking sector have diagonal form because of non-Abelian property of the family symmetry. In the present model, there are only three R-parity violating trilinear interactions allowed by the family symmetry, and baryon number violating terms $\lambda_{i j k}^{\prime \prime} U_{i}^{c} D_{j}^{c} D_{k}^{c}$ are forbidden by the symmetry. It should be compared with the MSSM case in which there are 45 trilinear couplings. These interactions can generate a lot of new processes which have not been observed yet such as lepton flavor violating (LFV) processes, or new contributions to already observed processes. Many authors have studied phenomenology of R-parity violation and obtained constraints on each coupling constant corresponding to each process in the MSSM case. b

In this section, we obtain constraints on the coupling constants $\lambda, \lambda_{1,2}^{\prime}$ at the weak scale. Since the various new processes generated by the interactions $W_{R}$ depend only on the three coupling constants, we can predict ratios of new processes independent of $\lambda \mathrm{s}$. We will also find the ratios of the LFV processes in this section. In the following analysis, we assume that the R-parity violating couplings $\lambda$ and $\lambda_{1,2}^{\prime}$ are real and positive $c$.

\subsection{Constraint on $\lambda$}

In this subsection, we consider the constraint on $\lambda L_{3} L_{I} E_{I}^{c}$ operator. The most stringent constraint on $\lambda$ is obtained from both $\mu \rightarrow$ eee and neutrinoless double

${ }^{\mathrm{b}}$ See Ref. 9135 and references therein.

${ }^{\mathrm{c}} \mathrm{CP}$ violation induced by the R-parity violating trilinear couplings in the soft SUSY breaking sector has been studied in Ref. 37 . 

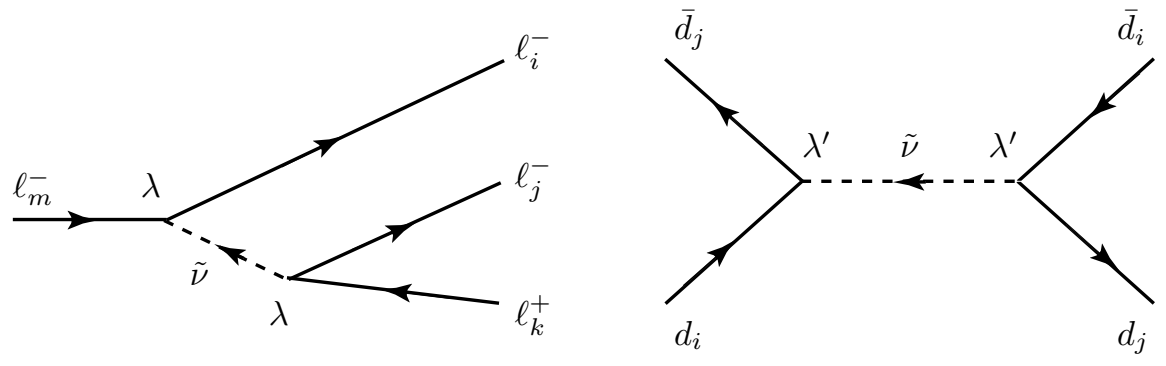

Fig. 1. Contributions to the decay processes $\ell_{m}^{-} \rightarrow \ell_{i}^{-} \ell_{j}^{-} \ell_{k}^{+}$by coupling $\lambda$ (left) and the neutral meson mixing by $\lambda^{\prime}$ (right).

beta decay, both the processes give similar bound. Here we explicitly show only $\mu \rightarrow$ eee process $11|12| 13|14| 32$. The decay process $\ell_{m}^{-} \rightarrow \ell_{i}^{-} \ell_{j}^{-} \ell_{k}^{+}$is generated by tree-level $t$ - and $u$ - channel sneutrino exchange (Fig. 1), and its effective Lagrangian is given by

$$
\mathcal{L}_{e f f}=\lambda^{2} A_{L}\left(\bar{\ell}_{i} P_{L} \ell_{m}\right)\left(\bar{\ell}_{j} P_{R} \ell_{k}\right)+\lambda^{2} A_{R}\left(\bar{\ell}_{i} P_{R} \ell_{m}\right)\left(\bar{\ell}_{j} P_{L} \ell_{k}\right)+(i \leftrightarrow j),
$$

where the coefficients are

$$
\begin{aligned}
A_{L}= & \frac{1}{m_{\tilde{\ell} 1 L}^{2}}\left(U_{e R}^{\dagger}\right)_{i J}\left(U_{e R}\right)_{J k}\left(U_{e L}^{\dagger}\right)_{j 3}\left(U_{e L}\right)_{3 m} \\
& +\frac{1}{m_{\tilde{\ell} 3 L}^{2}}\left(U_{e R}^{\dagger}\right)_{i J}\left(U_{e L}\right)_{J m}\left(U_{e L}^{\dagger}\right)_{j K}\left(U_{e R}\right)_{K k}, \\
A_{R}= & \frac{1}{m_{\tilde{\ell} 1 L}^{2}}\left(U_{e R}^{\dagger}\right)_{j J}\left(U_{e R}\right)_{J m}\left(U_{e L}^{\dagger}\right)_{i 3}\left(U_{e L}\right)_{3 k} \\
& +\frac{1}{m_{\tilde{\ell} 3 L}^{2}}\left(U_{e R}^{\dagger}\right)_{j J}\left(U_{e L}\right)_{J k}\left(U_{e L}^{\dagger}\right)_{i K}\left(U_{e R}\right)_{K m},
\end{aligned}
$$

with mixing matrices in Eq. (10). In our approximation, sneutrino mass is the same as that of the left-handed slepton. From this Lagrangian, the branching ratio of $\mu \rightarrow$ eee is given by,

$$
B R(\mu \rightarrow e e e)=\frac{4 \lambda^{4}}{64 G_{F}^{2}}\left[\left|A_{L}\right|^{2}+\left|A_{R}\right|^{2}\right] B R\left(\mu \rightarrow e \bar{\nu}_{e} \nu_{\mu}\right) .
$$

The requirement that this branching ratio should not exceed the experimental bound $B R(\mu \rightarrow e e e)^{e x p}<1.0 \times 10^{-12}$ provides a constraint on $\lambda$

$$
\lambda<1.4 \times 10^{-2}\left(\frac{m_{\tilde{\ell} L}}{100 \mathrm{GeV}}\right),
$$

where we have assumed $m_{\tilde{\ell} 1 L}=m_{\tilde{\ell} 3 L} \equiv m_{\tilde{\ell} L}$ in order to forbid the contribution to FCNC processes from the soft scalar mass terms. 


\subsection{Constraints on $\lambda_{1}^{\prime}$ and $\lambda_{2}^{\prime}$}

Constraints on $\lambda_{1,2}^{\prime}$ are obtained from neutral meson mixings 21|22|23|24|25|26|32, and on the products $\lambda \lambda_{1}^{\prime}$ and $\lambda \lambda_{2}^{\prime}$ from leptonic decays of neutral mesons $23|27 / 28| 29 \mid 32$. Since both processes are generated at tree level, these give the most stringent bounds on $\lambda_{1,2}^{\prime}$. Although $\mu-e$ conversion in nuclei $\frac{14[15]}{15}$ is also generated at tree level by $\lambda_{1,2}^{\prime}$, bounds from this process are weaker than those from neutral meson system.

The neutral meson mixing is generated at the tree level through the exchange of a sneutrino in both $s$ - and $t$ - channels (Fig. (1). For $K^{0}-\bar{K}^{0}$ mixing, the effective Hamiltonian is obtained as

$$
\mathcal{H}_{e f f}=\frac{\Lambda_{I 21}^{\prime} \Lambda_{I 12}^{\prime *}}{m_{\tilde{\ell} 1 L}^{2}}\left(\bar{d}_{R} s_{L}\right)\left(\bar{d}_{L} s_{R}\right)
$$

where

$$
\Lambda_{I j k}^{\prime}=\lambda_{1}^{\prime}\left(U_{d R}^{\dagger}\right)_{j J}\left(U_{d L}\right)_{3 k}\left(i \sigma^{2}\right)_{I J}+\lambda_{2}^{\prime}\left(U_{d R}^{\dagger}\right)_{j 3}\left(U_{d L}\right)_{J k}\left(\sigma^{1}\right)_{I J} .
$$

We require that these additional contributions to the mass difference of neutral $\mathrm{K}$ meson are smaller than its experimental value: The assumptions $\theta_{1}^{d}-\theta_{3}^{d}=0$ and $\lambda_{1}^{\prime}=\lambda_{2}^{\prime}$ lead to the most stringent constraints on $\lambda_{1,2}^{\prime}$ :

$$
\lambda_{1}^{\prime}=\lambda_{2}^{\prime}<3.1 \times 10^{-3}\left(\frac{m_{\tilde{\ell} L}}{100 \mathrm{GeV}}\right) .
$$

From leptonic decays of neutral Kaon, we get constraints on $\lambda \lambda_{1,2}^{\prime}$, which are

$$
\lambda \lambda_{1}^{\prime}<5.4 \times 10^{-7}\left(\frac{m_{\tilde{\ell} L}}{100 \mathrm{GeV}}\right)^{2}
$$

from $K_{L} \rightarrow \mu^{\mp} e^{ \pm}$, and

$$
\lambda \lambda_{2}^{\prime}<1.1 \times 10^{-8}\left(\frac{m_{\tilde{\ell} L}}{100 \mathrm{GeV}}\right)^{2}
$$

from $K_{L} \rightarrow e^{-} e^{+}$.

\subsection{Predictions for Lepton Flavor Violating processes}

Although many processes can be generated by the R-parity violating interactions, we focus on the LFV decays $\ell_{m}^{-} \rightarrow \ell_{i}^{-} \ell_{j}^{-} \ell_{k}^{+}$, where $m=\mu$ or $\tau$, in this subsection. As mentioned in the subsection 3.1, the operator $\lambda L_{3} L_{I} E_{I}^{c}$ generates the decays $\ell_{m}^{-} \rightarrow \ell_{i}^{-} \ell_{j}^{-} \ell_{k}^{+}$at tree level when $\lambda \neq 0$. The other two operators in Eq.(12), $\lambda_{1,2}^{\prime} L Q D^{c}$, also generate the similar decay processes at one loop level through photon penguin diagrams shown in Fig. 2, but we found that the bounds on $\lambda_{1,2}^{\prime}$ are stronger than that on $\lambda$ in the previous subsections. So we neglect contributions from $\lambda_{1,2}^{\prime}$ operators to the decays $\ell_{m}^{-} \rightarrow \ell_{i}^{-} \ell_{j}^{-} \ell_{k}^{+}$. Moreover, flavor changing $Z$ boson decay $Z \rightarrow \ell_{i}^{-} \ell_{j}^{+}$induced by the R-parity violating bilinear terms can contribute to $\ell_{m}^{-} \rightarrow \ell_{i}^{-} \ell_{j}^{-} \ell_{k}^{+}$processes. Since branching ratios of these decays are propotional 
to $\sin ^{2} \xi$, their effects are also negligible 20 . Besides these R-parity violating contributions, there are two other contributions to these processes by Higgs bosons. Since the charged leptons couple to the neutral Higgs bosons, these Yukawa interactions generate LFV processes at one loop level 38 . However, these effects are enhanced only when $\tan \beta$ is large. So, we assume that these are negligible because $\tan \beta$ is small enough. Moreover, since there are three generations of both up and down type Higgs doublet in this model, LFV processes mediated by the neutral Higgs bosons are generated at tree level (Fig. 2). However, the branching ratio of the $\mu \rightarrow$ eee from these effects is $B R \sim 10^{-16}$ when the neutral Higgs boson mass is $100 \mathrm{GeV}$ because of the smallness of the Yukawa couplings. So, these contributions can also be negligible compared to those from $\lambda L L E^{c}$ couplings unless $\lambda<10^{-3}$. Therefore, we can approximate that $\ell_{m}^{-} \rightarrow \ell_{i}^{-} \ell_{j}^{-} \ell_{k}^{+}$processes are induced at tree level only by $\lambda$. In this approximation, the ratios of these processes are independent of $\lambda$, but depend on the mixing matrices $U_{e L(R)}$ which reflect the flavor structure of the model. Therefore we find some predictions of LFV decays $\ell_{m}^{-} \rightarrow \ell_{i}^{-} \ell_{j}^{-} \ell_{k}^{+}$in our model.

From the branching ratio Eq.(16), we can easily find the ratios of processes in the approximation that all scalar masses are equald $\mathrm{d}$

$$
\begin{aligned}
\frac{B R(\tau \rightarrow e e e)}{B R(\tau \rightarrow \mu \mu \mu)} & \simeq \frac{4 \epsilon_{\mu}^{2}}{1+\epsilon_{\mu}^{2}}=0.014 \\
\frac{B R(\tau \rightarrow \mu \mu e)}{B R(\tau \rightarrow \mu \mu \mu)} & \simeq \frac{1-\epsilon_{\mu}^{2}+2 \epsilon_{e}^{2}}{1+\epsilon_{\mu}^{2}-2 \epsilon_{e}^{2}}=0.99 \\
\frac{B R(\mu \rightarrow e e e)}{B R(\tau \rightarrow e e e)} & \simeq \frac{\tau_{\mu}}{\tau_{\tau}} \epsilon_{\mu}^{5} \frac{\epsilon_{e}^{2}}{2 \epsilon_{\mu}^{2}+\epsilon_{e}^{2}}=0.0093
\end{aligned}
$$

where small parameters $\epsilon_{e, \mu}$ are given in Eq.(11) and $\tau_{\mu}\left(\tau_{\tau}\right)$ stand for the lifetime of the $\mu(\tau)$ lepton. Also, $B R(\tau \rightarrow \mu \mu e)$ means $B R\left(\tau^{-} \rightarrow \mu^{-} \mu^{-} e^{+}\right)$, and similar for the other processes. One can obtain the ratios of other combinations from the

\footnotetext{
${ }^{\mathrm{d}}$ From the conditions to suppress $\mu \rightarrow e+\gamma$ process from the scalar mass terms, slepton masses are required to be degenerated with mass differences of order $10^{-13}$.
} 

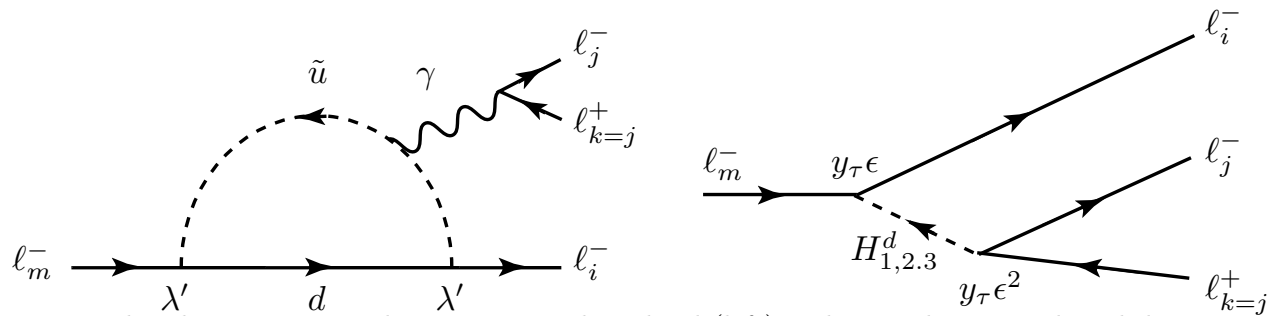

Fig. 2. The photon penguin diagram at one loop level (left) and Higgs boson mediated diagram at tree level (right) to the decays $\ell_{m}^{-} \rightarrow \ell_{i}^{-} \ell_{j}^{-} \ell_{k=j}^{+}$. These contributions can be negligible compared to the tree level processes induced by the coupling $\lambda$.

branching ratios listed below:

$$
\begin{aligned}
B R(\mu \rightarrow e e e) & \propto \tau_{\mu} \epsilon_{e}^{2}\left[2\left(1-2 \epsilon_{\mu}^{2}\right) m_{\tilde{\ell} 1 L}^{-4}+\frac{1}{2} m_{\tilde{\ell} 3 L}^{-4}-2\left(1-\epsilon_{\mu}^{2}\right) m_{\tilde{\ell} 1 L}^{-2} m_{\tilde{\ell} 3 L}^{-2}\right] \\
B R(\tau \rightarrow e e e) & \propto \tau_{\tau}\left[\epsilon_{\mu}^{2}\left(1-\epsilon_{\mu}^{2}-4 \epsilon_{e}^{2}\right) m_{\tilde{\ell} 1 L}^{-4}+\frac{1}{2} \epsilon_{e}^{2} m_{\tilde{\ell} 3 L}^{-4}\right] \\
B R(\tau \rightarrow \mu \mu \mu) & \propto \tau_{\tau}\left[\frac{1}{4}\left(1+\epsilon_{\mu}^{2}-2 \epsilon_{e}^{2}-4 \epsilon_{e}^{2} \epsilon_{\mu}^{2}\right) m_{\tilde{\ell} 3 L}^{-4}-2 \epsilon_{e}^{2} \epsilon_{\mu}^{2} m_{\tilde{\ell} 1 L}^{-2} m_{\tilde{\ell} 3 L}^{-2}\right] \\
B R(\tau \rightarrow e e \mu) & \propto \tau_{\tau} \epsilon_{e}^{2}\left[2 \epsilon_{\mu}^{2} m_{\tilde{\ell} 1 L}^{-4}+\left(\frac{1}{2}-\epsilon_{\mu}^{2}\right) m_{\tilde{\ell} 3 L}^{-4}-2 \epsilon_{\mu}^{2} m_{\tilde{\ell} 1 L}^{-2} m_{\tilde{\ell} 3 L}^{-2}\right] \\
B R(\tau \rightarrow \mu \mu e) & \propto \tau_{\tau} \frac{1}{4}\left(1-\epsilon_{\mu}^{2}+2 \epsilon_{e}^{2}-4 \epsilon_{e}^{2} \epsilon_{\mu}^{2}+\frac{1}{4} \epsilon_{\mu}^{4}\right) m_{\tilde{\ell} 3 L}^{-4}, \\
B R(\tau \rightarrow \mu e e) & \propto \tau_{\tau}\left[\epsilon_{e}^{2} \epsilon_{\mu}^{2} m_{\tilde{\ell} 1 L}^{-4}+\frac{1}{8}\left(1-2 \epsilon_{\mu}^{2}+6 \epsilon_{e}^{2} \epsilon_{\mu}^{2}+\frac{3}{2} \epsilon_{\mu}^{4}\right) m_{\tilde{\ell} 3 L}^{-4}\right] \\
B R(\tau \rightarrow \mu e \mu) & \propto \tau_{\tau}\left[\frac{1}{8}\left(1-4 \epsilon_{e}^{2}+6 \epsilon_{e}^{2} \epsilon_{\mu}^{2}-\frac{3}{4} \epsilon_{\mu}^{4}\right) m_{\tilde{\ell} 3 L}^{-4}+2 \epsilon_{e}^{2} \epsilon_{\mu}^{2} m_{\tilde{\ell} 1 L}^{-2} m_{\tilde{\ell} 3 L}^{-2}\right],
\end{aligned}
$$

where the common factor is not shown explicitly.

\section{Conclusion}

We have considered the properties of R-parity violating operators in a SUSY model with non-Abelian discrete $Q_{6}$ family symmetry. The family symmetry can reduce the number of parameters in the Yukawa sector, and explain the fermion masses and mixings between generations. It can also reduce the number of R-parity violating couplings and determine the form of those. Only three trilinear couplings are allowed, and the baryon number violating operators are forbidden by the symmetry in our model. We derived upper bounds on these couplings: $\lambda<O\left(10^{-2}\right)$, $\lambda_{1,2}^{\prime}<O\left(10^{-3}\right)$, and obtained the predictions on the ratios of the LFV decays $\ell_{m}^{-} \rightarrow \ell_{i}^{-} \ell_{j}^{-} \ell_{k}^{+}$which do not depend unknown parameters. The results reflect the properties of the family symmetry because these predictions contain the mixing matrices of the charged lepton sector which is written by masses of the charged 
leptons. Our predictions can be testable at future experiments because the superB factory 39 or LHC 40 will have the sensitivity $B R \sim 10^{-(8-9)}$ for LFV $\tau$ decays.

\section{Acknowledgments}

The author would like to thank the organizers for invitation to the conference. This work is supported by the ESF grant No. 6190 and postdoc contract 01-JD/06.

\section{References}

1. Y. Kajiyama, JHEP 0704007 (2007).

2. K. S. Babu and J. Kubo, Phys. Rev. D71, 056006 (2005).

3. E. Itou, Y. Kajiyama and J. Kubo, Nucl. Phys. B743, 74 (2006).

4. J. Kubo, A. Mondragón, M. Mondragón and E. Rodríguez-Jáuregui, Prog. Theor. Phys. 109, 795 (2003); Erratum-ibid.114, 287 (2005); J. Kubo, Phys. Lett. B578, 156 (2004); Erratum-ibid. B619, 387 (2005); Y. Kajiyama, J. Kubo and H. Okada, Phys. Rev. D75, 033001 (2007).

5. T. Kobayashi, J. Kubo and H. Terao, Phys. Lett. B568, 83 (2003); K-Y. Choi, Y. Kajiyama, H. M. Lee and J. Kubo, Phys. Rev. D70, 055004 (2004).

6. A. Abulencia et al. [CDF collaboration], Phys. Rev. Lett. 97, 062003 (2006).

7. P. Minkowski, Phys. Lett. B67, 421 (1977). T. Yanagida, in Proc, of the Workshop on the unified Theory and Baryon Number in the universe, ed. O. Sawada and A. Sugamoto, (KEK report 79-18, 1979); M. Gell-Mann, P. Ramond and R. Slansky, in Supergravity, ed P. van Nieuwenhuizen and d.Z. Freedman, (North Holland, Amsterdam, 1979); R. N. Mohapatra and G. Senjanovic, Phys. Rev. Lett. 44, 912 (1980).

8. G. R. Farrar and P. Fayet, Phys. Lett. B76, 575 (1978).

9. G. Bhattacharyya, Nucl. Phys. Proc. Suppl. 52A, 83 (1997); H. K. Dreiner, hep-ph/9707435 P. Roy, hep-ph/9712520 M. Chemtob, Prog. Part. Nucl. Phys. 54, 71 (2005); R. Barbier et al. Phys. Rept. 420, 1 (2005).

10. L. E. Ibanez and G. G. Ross, Nucl. Phys. B368,3 (1992).

11. L. J. Hall and M. Suzuki, Nucl. Phys. B231, 419 (1984);

12. S. Dawson, Nucl. Phys. B261, 297 (1985); V. Barger, G. F. Giudice and T. Han, Phys. Rev. D40, 2987 (1989).

13. I. Hinchliffe and T. Kaeding, Phys. Rev. D47, 279 (1993).

14. A. de Gouvêa, S. Lola and K. Tobe, Phys. Rev. D63, 035004 (2001).

15. J. E. Kim, P. Ko and D-G. Lee, Phys. Rev. D56, 100 (1997); K. Huitu, J. Maalampi, M. Raidal and A. Santamaria, Phys. Lett. B430, 355 (1998).

16. M. Chaichian and K. Huitu, Phys. Lett. B384, 157 (1996); B. de Carlos and P. L. White, Phys. Rev. D54, 3427 (1996).

17. R. N. Mohapatra, Phys. Rev. D34, 3457 (1986); S. Dimopoulos and L. J. Hall, Phys. Lett. B207, 210 (1987); K. S. Babu and R. N. Mohapatra, Phys. Rev. Lett. 64, 1705 (1990); G. Bhattacharyya and H. V. Klapdor-Kleingrothaus and H. Päs, Phys. Lett. B463, 77 (1999).

18. A. S. Joshipura and M. Nowakowski, Phys. Rev. D51, 2421 (1995); R. Hempfling, Nucl. Phys. B478, 3 (1996).

19. T. Banks, Y. Grossman, E. Nardi and Y. Nir, Phys. Rev. D52, 5319 (1995); P. Binétruy, E. Dudas, S. Lavignac and C. A. Savoy, Phys. Lett. B422, 171 (1998).

20. M. Bisset, O. C. W. Kong, C. Macesanu and L. H. Orr, Phys. Lett. B430, 274 (1998).

21. R. Barbieri and A. Masiero, Nucl. Phys. B267, 679 (1986).

22. B. de Carlos and P. L. White, Phys. Rev. D55, 4222 (1997). 
23. G. Bhattacharyya and A. Raychaudhuri, Phys. Rev. D57, 3837 (1998).

24. J. P. Saha and A. Kundu, Phys. Rev. D69, 016004 (2004).

25. A. Kundu and J. P. Saha, Phys. Rev. D70, 096002 (2004).

26. R. M. Wang, G. R. Lu, E. K. Wang and Y. D. Yang, hep-ph/0609276.

27. Y. Grossman, Z. Ligeti and E. Nardi, Phys. Rev. D55, 2768 (1997).

28. J-H. Jang, J. K. Kim and J. S. Lee, Phys. Rev. D55, 7296 (1997); S. Baek and Y. Kim, Phys. Rev. D66, 077701 (1999); J. P. Saha and A. Kundu, Phys. Rev. D66, 054021 (2002).

29. H. Dreiner, G. Polesello and M. Thormeier, Phys. Rev. D65, 115006 (2002).

30. V. Ben-Hamo and Y. Nir, Phys. Lett. B339, 77 (1994).

31. J. L. Goity and M. Sher, Phys. Lett. B346, 69 (1995); Erratum-ibid, B385, 500 (1996); C. E. Carlson, P. Roy and M. Sher, Phys. Lett. B357, 99 (1995); J. Hisano, hep-ph/0004266 F. Vissani, Phys. Rev. D52, 4245 (1995); A. Y. Smirnov and F. Vissani, Phys. Lett. B380, 317 (1996); G. Bhattacharyya and P. B. Pal, Phys. Rev. D59, 097701 (1999); Phys. Lett. B439, 81 (1998).

32. D. Choudhury and P. Roy, Phys. Lett. B378,153 (1996); K. Agashe and M. Graesser, Phys. Rev. D54, 4445 (1996).

33. A. H. Chamseddine and H. Dreiner, Nucl. Phys. B458, 65 (1996); P. Binétruy, S. Lavignac and P. Ramond, Nucl. Phys. B477, 353 (1996); E. J. Chun and A. Lukas, Phys,. Lett. B387, 99 (1996); J. Ellis, S. Lola and G. G. Ross, Nucl. Phys. 526, 115 (1998); G. Eyal and Y. Nir, JHEP. 9906, 024 (1999); A. S. Joshipura, R. Vaidya and S. K. Vempati, Phys. Rev. D62, 093020 (2000).

34. C. D. Carone, L. J. Hall and H. Murayama, Phys. Rev. D54, 2328 (1996); G. Bhattacharyya, Phys. Rev. D57, 3944 (1998).

35. B. C. Allanach, A. Dedes and H. K. Dreiner, Phys. Rev. D60, 075014 (1999).

36. M. Frigerio, S. Kaneko, E. Ma and M. Tanimoto, Phys. Rev. D71, 011901 (2005).

37. S. A. Abel, Phys. Lett. B410, 173 (1997).

38. K. S. Babu and C. Kolda, Phys. Rev. Lett. 89, 241802 (2002).

39. A. G. Akeroyd et al. [SuperKEKB Physics Working Group], KEK report 04-4, arXiv:hep-ex/0406071.

40. R. Santinelli and M. Biasini, CMS-note/2002-037; N. G. Unel, hep-ex/0505030 